\title{
TESTE DE TUBERCULIZAÇÃO EM OVINOS (OVIS ARIES) EXPERIMENTALMENTE SENSIBILIZADOS
}

\author{
F.C. Cyrillo', M .L. do R. Leal'2, A. M oreno'1, P.M .P.C. M otta3, \\ I.L. Sinhorini ${ }^{4}$, S.A. V asconcellos 5 , S.R. Pinheiro' ${ }^{5}$, F.J. Benesi ${ }^{1}$
}

IUniversidadedeSão Paulo, FaculdadedeM edicinaVeterináriaeZootecnia, Departamento deClínicaM édica, Av.Prof.Dr. Orlando Marques dePaiva, 87, CEP 05508-900, São Paulo, SP, Brasil. E-mail: fercyrillo@uol.com.br

\begin{abstract}
RESUMO
Esta pesquisa visou estabelecer valores de referência para a interpretação da reação tuberculínica em ovinos experimentalmente inoculados com cepas padrões de $M$ ycobacterium bovis $\mathrm{AN}_{5}$ eM ycobacterium avium $\mathrm{D}_{4}$, além de avaliar possíveis reações clínicas provocadas pela resposta imuno-alérgica intradérmica à tuberculina. Utilizou-se 30 ovinos, distribuídos por 3 grupos de 10 animais cada, a saber: A - sensibilizados M . avium; B - sensibilizados M . bovis e C - não sensibilizado (controle) - que receberam injeção de solução fisiológica. A pós 45 dias da sensibilização, realizou-se o Teste Cervical Comparativo (TCC) com reação medida pela variação da espessura da pele com cutímetro de mola, nos seguintes momentos: antes da aplicação das tuberculinas (PPDs -derivado protéi co purificado), 12h, 24h, 48h, 72h e 96h após a aplicação. Em relação à reação ao PPD bovino, no TesteCervical Simples (TCS), lido às 72h ( \pm 6 horas) pós-tuberculinização (p. t.), considerou-se uma reação positiva, quando o aumento da espessura da pele foi igual ou mai or que $2,40 \mathrm{~mm}$; como inconclusiva, com aumento entre 1,00 e 2,40 mm, e como reação negativa, aumentos da espessura da pele inferiores a $1 \mathrm{~mm}$. A anál ise dos resultad os da leitura do TCC, realizada às $72 \mathrm{~h}$ p. t., permitiu concluir queo animal poderá ser consi derado com resposta: positiva, quando a reação ao PPD bovino superar aquela ao aviário em pelo menos $2 \mathrm{~mm}$; inconclusiva, quando a reação ao PPD bovino for maior que aquel a ao aviário, com diferença entre 1 e1,90 mm, enegativa quando a reação bovinafor menor que a aviária ou maior em até $0,90 \mathrm{~mm}$. Estes val ores obtidos para o TCS eTCC são propostos como padrão de interpretação desses testes em ovinos.
\end{abstract}

PALAVRAS-CHAVE: Tuberculose, diagnóstico, Teste Cervical Comparativo, Teste Cervical Simples, ovinos.

\section{ABSTRACT}

TUBERCULIN TEST IN EXPERIMENTALLY SENSITIZED SHEEP (OVIS A RIES). This study aimed to establish the reference values for the interpretation of the skin test in sheep experimentally inoculated with standard strains of $\mathrm{M}$ ycobacterium bovis $\mathrm{AN}_{5}$ and $\mathrm{M}$ ycobacterium avium $\mathrm{D}_{4}$, and evaluated theclinical manifestationsinduced by theintradermicimmune-allergic responseto tuberculin. Thirty healthy sheep were separated in threegroups: $A$ ) sensitized with $M$. avium, $n=10 ; B$ ) sensitized with $M$. bovis, $n=10$; and $C$ ) control, $n=10$, which were injected with saline solution. After 45 days of inoculation, the Comparative Cervical Test (CCT) was evaluated by the variation of dermal thickness measured by skinfold cal ipers carried out at the following moments: beforetheadministration of tuberculin (PPD - purified protein derivative) and at $12,48,72$ and 96 hours after PPD inoculation. In regard to the bovine PPD, the Single Cervical Test (SCT), observed at 72 hours ( \pm 6 hours) post-tuberculinization (p.t.) the response was considered as: positive, when skinfold thickness was equal or greater than $2.40 \mathrm{~mm}$; inconclusive, when skinfold thickness was between 1 and $2.40 \mathrm{~mm}$; and negative, when skinfold thickness was lower than $1 \mathrm{~mm}$. Analyses of the results of the CCT performed at $72 \mathrm{hr}$ p.t.

\footnotetext{
${ }^{2}$ Universidade de Santa Maria, Departamento de Grandes Animais, Santa Maria, RS, Brasil.

${ }^{3}$ Laboratório de Referência A nimal (LARA) - Pedro Leopoldo, MG, Brasil.

${ }^{4}$ Universidade de São Paulo, Faculdade de Medicina Veterinária eZootecnia, Departamento de Patologia, São Paulo, SP, Brasil.

5Universidade de São Paulo, Faculdade de M edicina Veterinária e Zootecnia, Departamento de M edicina Veterinária Preventiva e Saúde A nimal, São Paulo, SP, Brasil.
} 
indicated that theanimal would beconsidered as: positive, when the bovinePPD responsewas greater than the avian by at least $2.00 \mathrm{~mm}$; inconclusive, when the PPD bovine reaction was greater than theavian variation by between 1 and $1.90 \mathrm{~mm}$, and negative, when theresponse to the bovine PPD was less than the avian or greater by up to $0.90 \mathrm{~mm}$. These values obtained for SCT and CCT are proposed as reference values for tests in sheep.

KEY WORDS: Tuberculosis, diagnostic, ComparativeCervical Tests, SingleCervical Test, sheep.

\section{INTRODUÇÃO}

A tuberculose foi considerada por muito tempo rara nos ovinos, acreditando-se na resistência natural dessa espécie à infecção causada pelo M ycobacterium SSP. (BARTON; ACLAND, 1973; CoRDES et al., 1981; Craig; Davies, 1938; Creech, 1940; Hiepe, 1972; LUKE, 1958; RAdostits et al., 2002; THOEN, 1988). Estefato podeser atribuído a diversos fatores, sendo um deles o sistema de manejo ao qual as diferentes espécies de ruminantes estão submetidas (GRIFFITH, 1928; J ENSEN; SWIFT, 1982). Os ovinos podem adquirir aenfermi dadequandoem contato comanimais doentes, sejam eles bovinos, bubalinos, caprinos ou animais silvestres (AlLEN, 1988; DAVIDSON et al.,1981; FOULERTON, 1902; M'FADYEAN, 1902).

A tuberculose ovina, quanto aos agentes causais e sua patogenicidade, à forma clínica de apresentação, freqüência, aspectos epidemiológicos e zoonóticos, apresenta uma grande semel hança à tuberculose bovina HIEPE, 1972; JENSEN; SWIFT, 1982; MALONE et al., 2003; M ARSH, 1965). Hárelatos da ocorrência da enfermidade em ovinos em países como EUA, Inglaterra, N ovaZelândia, Á frica, Irlanda, Alemanha e Brasil, sendo o diagnóstico da tuberculose realizado pelo achado delesõestípicas emnecropsias ou análises histopatológi cas, isolamento bacteriológico, em levantamentos da doença em inspeção nos abatedourosepor meio do testetuberculínico(BARTON; ACland, 1973; BenesI, et al., 2006; Cordes et al., 1981; Craig; Davies, 1938; Creech, 1940; D avidson et al., 1981; LUKE, 1958; MARSH, 1965; M'FAdYEAN, 1902; MURPHY, 1935; WhITTY; DEM PSEY, 1974).

A pecuária destinada à criação de ovinos vem se expandindo há muito tempo e diversificando a sua exploração. Em condições criatórias brasileiras, antigamente os ovinos eram utilizados apenas para a subsistência familiar, particularmente para produção de lã e carne (BRITo, 2004). Com a evolução da seleção genética e o desenvolvimento tecnológico percebeu-se que esta espécie poderia ser uma fonte valiosaderenda, não só pela comercial ização deseus produtos tradicionais, mas também pela venda do leite e seus subprodutos (BencinI; Pulina, 1997; BRIto et al., 2006; Peeters et al., 1992).

Para o controle e erradicação da tuberculose animal, deveriadestacar-senãosóosbovinosebubalinos, mas também a importância da realização do teste tuberculínico em outras espécies animais de produ- ção, como caprinos e ovinos, além dos ruminantes silvestres, pois estes espécimes poderiam atuar como fontedeinfecção datuberculoseparaosbovinos. Esta ação evitaria também que seres humanos, consumidores de seus produtos e subprodutos, fossem afetados pela enfermidade (Antunes et al., 2002).

A legislação nacional pertinente ao Programa Nacional deSanidadedeCaprinoseOvinos (PNSCO) ainda não está concluída para implantação, mas já existe, por parte dos técnicos da área e criadores, a solicitação de quea tubercul ose seja inserida no programa(BRASIL, 2004b). Em qualquer situação ressaltaria a necessidade de se determinar os valores de referênciapara otestedetuberculina em ovinos como ferramenta de programa específico para combate e erradicaçãodatuberculosenessaespécieanimal (BRASIL, 2004a).

Diante da precariedade de dados nacionais sobre a doença em ovinos, daimportância do temaeanecessidade deaprofundamento das técnicas dediagnóstico pertinenteao seu combateeerradicação, estetrabaIhofoi delineado paraavaliar arespostaimuno-alérgica cutânea à tuberculina em ovinos (Ovis aries).

\section{MATERIAL E MÉTODOS}

Utilizou-se 30 ovinos adultos clinicamente sadios, negativos ao Teste Cervical Comparativo (TCC), executado segundo as normas adotadas, no Brasil, para as espécies bovina e bubalina (BRASIL, 2004a).

Aos 45 dias do teste tuberculínico de triagem inicial, os ovinos foram distribuídos deforma homogênea, deacordo compeso eo sexo por 3 grupos, cada umcom dez animais, einoculadosviaintramuscular com $0,5 \mathrm{~mL}$ dos inóculos sensibilizantes com a concentração de $4 \mathrm{mg} / \mathrm{mL}$ de suspensão de $M$. avium amostra $\mathrm{D}_{4}$ morta pelo cal or e em veículo ol eoso (no grupo $A$ ) esuspensão deM . bovis amostra $A N_{5}$ morta pelo calor eem veículo ol eoso (no grupo B). O grupo controle(C), não sensibilizado, recebeu $0,5 \mathrm{~mL}$ de solução fisiológica. Os inóculos sensibilizantes padrões (partida 001/ 02) foram preparados pelo Laboratório de Referência Animal, do Ministério da A gricultura, Pecuária e A bastecimento - LARA - MAPA, Pedro Leopoldo, M inas Gerais, preparados segundo as recomendações do Centro Panamericano de Zoonoses (CPZ, 1980). Decorridos 35 dias póssensibilização, efetuou-se o Teste de Tuberculina 
Cervical comparativo emtodososanimais, injetandose $0,1 \mathrm{~mL}$ detuberculinaaviária $(0,5 \mathrm{mg} / \mathrm{mL})$ e0,1 $\mathrm{mL}$ de tuberculina bovina $(1 \mathrm{mg} / \mathrm{mL})$. A tuberculina aviária foi injetada cranialmente e a bovina caudalmente, na região cervical (tábua do pescoço), em dois pontos distantes entre si em $\pm 7 \mathrm{~cm}$, utilizando-se seringas tuberculínicas descartáveis calibradas. A espessura da dobra da pele foi medida com cutímetrodemola*, no momento imediatamenteanterior à aplicação das tubercul inas e decorridas 12, 24, 48, 72 e 96h pós tuberculizanção (p.t.).

Quanto à sua distribuição normal, os resultados obtidos foram analisados utilizando-se o teste de Kolmogorov-Smirnov; quantoàanálisedevariância, o testeF (ANOVA) e, quando esta análisefoi significativa, real izou-seum contrasteentremédiaspelotestede Duncan. O nível designificânciaadotad ofoi de $5 \%$. Os interval os de confiança (95\%) das mensurações das espessuras de pele, realizadas às $12,24,48,72$ e 96h, foram calculadosecomparadosentresi (SAMPAIO,1998), para avaliar as respostas homólogas, heterólogas e reações mecânicas. Utilizou-se o programa estatístico Graphpad Instat para a obtenção destes intervalos.

\section{RESULTADOSE DISCUSSÃO}

Duranteo experimento, os ovinos não apresentaram alterações clínicas notáveis no estado geral, com asfunções vitais (freqüência respiratória, batimentos cardíacos, movimentos ruminais etemperatura) permanecendo dentro dos limites fisiológicos de variação para a espécieovina (PugH, 2005). Essas observações concordaram com a descrição da reação à tuberculina como sendo caracterizada por uma resposta inflamatória no local de aplicação, estimulando uma reação dehipersensibilidadetardia (tipoIV), geralmente sem manifestações sistêmicas (TIZARD, 2002), além de reproduzirem os resultados obtidos por Silva et al. (2006) em caprinos.

Os resultados do TCC em ovinos sensibilizados experimental menteestão demonstrados na Tabela 1. Asreaçõestuberculínicasobtidascomouso dosPPDs bovino eaviário permitirama distinção entrereações homólogas e heterólogas (SILvA et al., 2006).

A reação tuberculínicamedidaatravés do aumento da espessura de peleatingiu maior magnitudeàs $48 \mathrm{~h}$ após ainjeção do PPD (Figs. 1e2); não havendo porém diferença estatística em relação à observada às $72 \mathrm{~h}$ ( \pm 6h) (Tabela 1). A evolução da intensidade da reação está em concordância com as afirmações de TIZARD (2002), quedestacam queos animaisinfectados porM . bovis apresentamumarespostainflamatóriaquepoderia atingir maior magnitude entre 24 a $72 \mathrm{~h}$ após o desencadeamento. De forma diversa, SAHOо (1951) e Roxo et al. (1998) para em seus trabal hos, respectivamente, com bovinos e bubalinos, determinaram quea reação apresentava uma maior intensidadeàs $72 \mathrm{~h}$. Da mesma forma que no presente estudo, SUTHER et al. (1974) não encontraram variação na resposta tuberculínicaem bovinos, observadasàs $48 \mathrm{e} 72 \mathrm{~h}$. SiLva et al. (2006), por sua vez, em seu estudo em caprinos, evidenciaram queo momento demai or intensidadefoi também constatado às $48 \mathrm{~h}$, esem diferença estatística em relação àquela observada às $72 \mathrm{~h}( \pm 6 \mathrm{~h})$. Devedestacar-seque, a maioria dos autores quetrabalhou com a espécie bovina, utiliza $72 \mathrm{~h}( \pm 6 \mathrm{~h})$ como momento padrão para a leitura do teste tuberculínico (DuFFIELD et al., 1985; GonzÁlez Llamazare et al., 1999; Lesslie; HeberTy, 1975; Ragassa; AmenI, 2001; Suther et al., 1974), e que o Ministério da Agricultura Pecuária e A bastecimento, através do PN CEBT, recomendaquea leitura do testetuberculínico, em bovinos ebubalinos, também seja realizada às $72 \mathrm{~h}( \pm 6 \mathrm{~h})$ após a aplicação da tuberculina (BRASIL, 2004a).

Em relação aos ovinos, o momento ideal para a leitura dareação àtuberculina, conformealiteratura, obedece a mesma tendência de realização às $72 \mathrm{~h}$, considerando-se os trabalhos de Eobinson (1955), DAVIDSON et al. (1981)eMALONE et al. (2003), enquanto, HIEPE (1972) e CoRDEs et al. (1981) adotavam como momento padrão de leitura o período de $72 \mathrm{~h}$ às $96 \mathrm{~h}$. Osresultadosobtidosnesteestudo, por apresentarem valores semel hantes estatisticamente às 48 e $72 \mathrm{~h}$, permitemaadoção doúltimotempo comoaqueleaser usado para a leitura do TCC (BRASIL, 2004a).

NoBrasil, alinfadenitecaseosadosovinos, causada por Corynebacterium pseudotuberculosis, éimportante para os ovinocultores, pois está disseminada nas criações devendo ser diferenciada da tuberculose, visto queRIET-CorRÊA et al. (2001), relataram uma alta prevalência de 50 a $60 \%$ da linfadenite caseosa em ovinos. Estefato reforça a importância da realização dotestetuberculínicocomo provaderotinaem peque nos ruminantes, por ter alta especificidade, com a finalidadederealizar-seo diagnóstico diferencial da linfadenite caseosa com a tuberculose.

O TCC, por sua vez, éutilizado para a eliminação de reações inespecíficas e possível confirmação de positividadedeanimaisqueapresentaramumareação inconclusiva ou positiva, ao PPD bovino, no teste intradérmico simples. No entanto, CoRDEs etal. (1981), em trabal ho realizado com ovinos, na N ova Zelândia, determinaram uma especificidade de 99,6\% e uma sensibilidade de $81,6 \%$, na utilização do teste intradérmicosimplesnaregião interna da coxa, considerando animais com aumento de espessura de pele superior a $2 \mathrm{~mm}$ como positivos para a tuberculose.

\footnotetext{
*Hauptner ${ }^{\circledR}$
} 
Tabela 1 - Média aritmética e desvio padrão $(\mathrm{mm})$ dos aumentos de espessura da pele de ovinos induzidos pelas tuberculinas aviária e bovina e as diferenças $(\mathrm{mm})$ entre a intensidade de reação às tuberculinas bovina e aviária, aplicadas na região cervical, segundo os grupos experimentais e os momentos da leitura dos resultados.

\begin{tabular}{|c|c|c|c|c|c|c|}
\hline \multirow[t]{2}{*}{ Grupo } & \multicolumn{6}{|c|}{ Tuberculina aviária } \\
\hline & Oh (a.i.) & 12h (p.i.) & 24h (p.i.) & 48h (p.i.) & 72h (p.i.) & 96h (p.i.) \\
\hline$A$ & $4,44 \pm 0,66$ aA & $5,84 \pm 0,50 a A B$ & $7,71 \pm 0,98 \mathrm{aBC}$ & $8,44 \pm 1,70 \mathrm{aC}$ & $7,49 \pm 0,92 \mathrm{aBC}$ & $6,84 \pm 1,13 \mathrm{aBC}$ \\
\hline $\mathrm{B}$ & $4,33 \pm 0,85$ aA & $5,24 \pm 0,80$ aA B & $6,39 \pm 1,02 \mathrm{aB}$ & $6,83 \pm 1,55 \mathrm{abB}$ & $6,54 \pm 1,92 \mathrm{aB}$ & $5,93 \pm 1,77$ aA \\
\hline \multirow[t]{2}{*}{$\mathrm{C}$} & $4,23 \pm 1,42 \mathrm{Aa}$ & $4,94 \pm 1,12 \mathrm{aA}$ & $5,89 \pm 1,53 a A$ & $5,74 \pm 1,26$ bA & $5,92+1,37 a A$ & $5,51 \pm 1,43 a A$ \\
\hline & \multicolumn{6}{|c|}{ Tuberculina bovina } \\
\hline$A$ & $4,90 \pm 1,09 \mathrm{aA}$ & $5,88 \pm 0,68 \mathrm{aA}$ & $6,66 \pm 0,87 \mathrm{aA}$ & $6,34 \pm 1,03 \mathrm{aA}$ & $6,36 \pm 1,09 \mathrm{aA}$ & $6,05 \pm 1,11 \mathrm{aA}$ \\
\hline $\mathrm{B}$ & $4,85+0,94 \mathrm{aA}$ & $6,26 \pm 0,76$ aA & $8,35 \pm 1,27 \mathrm{aBC}$ & $9,98 \pm 2,59 \mathrm{bc}$ & $9,64 \pm 3,20 \mathrm{bc}$ & $9,26 \pm 3,29 b c$ \\
\hline \multirow[t]{2}{*}{$\mathrm{C}$} & 4,86+1,81 aA & $5,08+1,65$ aA & $5,75+1,53$ aA & $5,33 \pm 1,48 \mathrm{aA}$ & $5,54 \pm 1,50 \mathrm{aA}$ & $5,34 \pm 1,37$ aA \\
\hline & \multicolumn{6}{|c|}{ Diferença PPD bovino e aviário } \\
\hline$A$ & $0,46 \pm 0,69 a A$ & $0,04 \pm 0,68 \mathrm{aA}$ & $1.05 \pm 0.79 \mathrm{aAB}$ & $2,1 \pm 1,71 \mathrm{aB}$ & $1,13+0,94 \mathrm{aA}$ & $0,79+0,76 \mathrm{aA}$ \\
\hline $\mathrm{B}$ & $0,52 \pm 0,50 \mathrm{aA}$ & $1,02 \pm 0,48 \mathrm{aA}$ & $1,96 \pm 1,12 \mathrm{bAB}$ & $3, \overline{15} \pm 2,02 \mathrm{bB}$ & $3,10 \pm 2,61 \mathrm{bB}$ & $3,33 \pm 2,61 \mathrm{bB}$ \\
\hline $\mathrm{C}$ & $0,63 \pm 0,79 a A$ & $0,14 \pm 0,75 a A$ & $0,14 \pm 0,69 a A$ & $0,41 \pm 0,75 a A$ & $0,38 \pm 0,97 a A$ & $0,17 \pm 0,77 a A$ \\
\hline
\end{tabular}

Nota: Médias comletras minúsculas diferentes, na mesma coluna, indicam diferença estatística entreosgrupos dentro do momento de leitura $(p<0,05)$.

Médias com letras maiúsculas diferentes, na mesma linha, indicam diferença estatística entre os momentos de leitura dentro de cada grupo $(p<0,05)$.

a.i. =diferença demedida da espessura da pele em mm, entre o local de aplicação do PPD bovino eo aviário, antes da injeção da tuberculina.

p.i. = diferença de medida da espessura da pele em mm, entre o local de aplicação do PPD bovino eo aviário, após a injeção da tuberculina.

Grupo A - sensibilizado (M . avium); Grupo B - sensibilizado (M . bovis) e Grupo C - não sensibilizado/ controle.

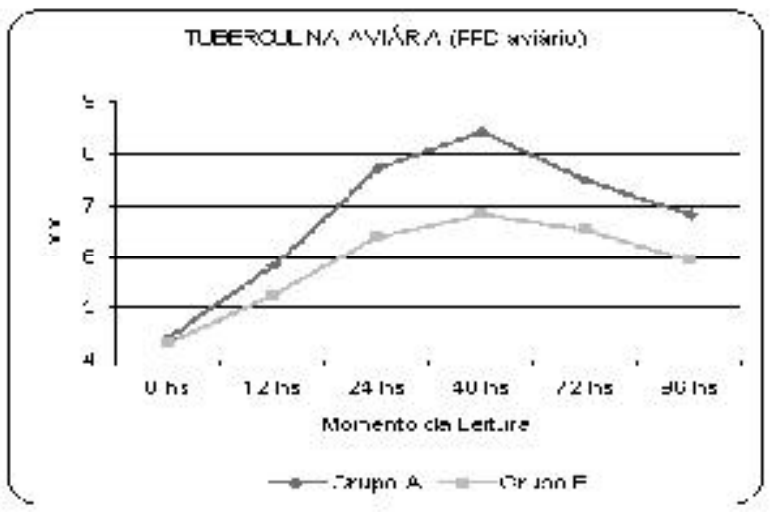

Fig 1 - Valores médios da espessura da pele de ovinos, medidos em milímetros, no local da aplicação do PPD aviário, segundo os grupos experimentais (Grupo A sensibilizado com $M$. avium; grupo B -sensibilizado com $M$. bovis) e o momento da leitura do resultado do teste cervical comparativo.

Considerando-se os valores mostradosnaTabela 1, com relação à reação ao PPD bovino, no teste intradérmico simples na região cervical, às 72 horas, pode-se interpretar para ovinos uma reação como:

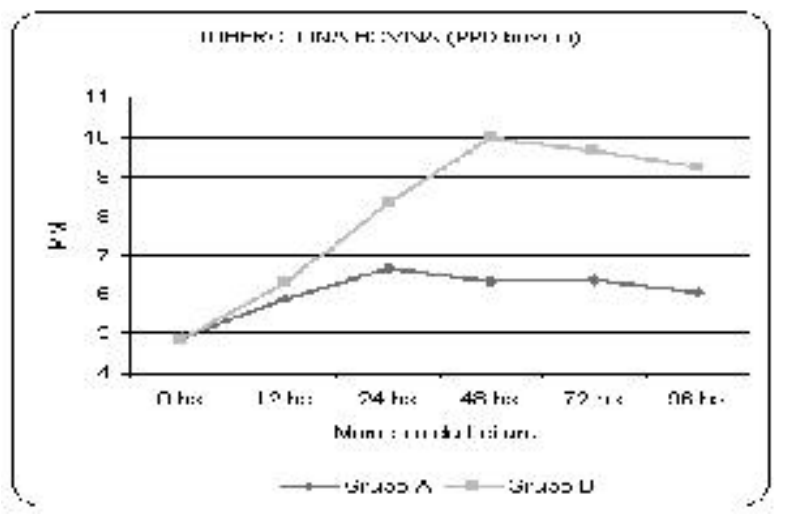

Fig. 2 - Valores médios da espessura da pele de ovinos, medidos em milímetros, no local de aplicação do PPD bovino, segundo os grupos experimentais (Grupo Asensibilizado com M . avium; grupo B-sensibilizado com $M$. bovis ) eo momento da leitura dos resultados do teste cervical comparativo.

negativa, coma observação deaumento deespessura de pele inferior a $1 \mathrm{~mm}$; inconclusiva, quando o aumento estiver entre 1 e $2,40 \mathrm{~mm}$, epositiva quando for maior ou igual a 2,40 mm. Estes valores recomenda- 


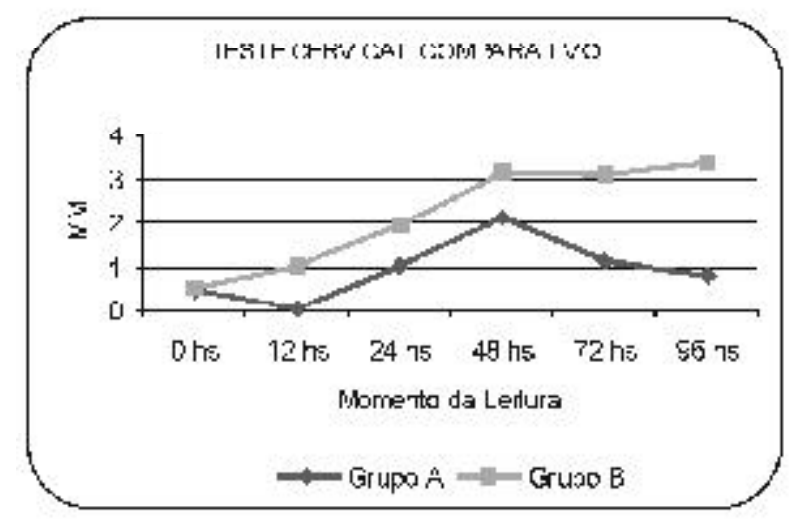

Fig.3-Valoresmédiosdadiferença entrea espessurada pele de ovinos, medidos em milímetros, nos locais de reação ao PPD bovino e ao PPD aviário, segundo os grupos experimentais (Grupo A - sensibilizado porM . avium; Grupo B - sensibilizado por M . bovis) eo momento da leitura dos resultados do testecervical comparativo.

dos para a interpretação dos resultados do teste intradérmico simples, na região cervical, são diferentes daqueles determinados por outros autores. Assim sendo, HIEPE (1972) usou a seguinte interpretação para o teste intradérmico simples na região cervical: negativo, sea espessura da pelefor inferior a 1,4 mm; inconclusivo, com aumento entre 1,5 a $3 \mathrm{~mm}$, e positivo quando o aumento for superior a $3 \mathrm{~mm}$. Por sua vez, CoRDEs et al. (1981) consideraramexclusivamente uma reação como positiva, quando a resposta for maior ou igual a $2 \mathrm{~mm}$. MALONE et al. (2003), com base em padrão estabelecido para bovinos, consideravam uma reação positiva, quando a resposta ao PPD bovino fosse superior ao aviário em pelo menos $5 \mathrm{~mm}$.

Essas diversidades de recomendações por si só val orizam o objetivo desta pesquisa e o acerto em se buscar o estabel ecimento depadrões deinterpretação particulares aos diferentes países, considerando as condições epidemiológicas em que são criados os animais, em especial manejo eambiente.

Preocupados com a questão da disseminação da tuberculoseemanimaisruminantes ecoma possibilidade de funcionarem como fonte de infecção para os bovinos, Roxo et al. (1998) estabel eceram um padrão de interpretação para o teste tuberculínico em búfalos e Si Lva et al. (2006), com o uso de caprinos experimentalmente sensi bilizados, propuseramvaIores limites padrão para a interpretação do teste tuberculínico. Em ruminantes silvestres, com o uso de um padrão estabelecido experimentalmente por CorRIn et al. (1987), MACKINTOSH et al. (2004) realizaram o testetuberculínico em cervos (Cervus el aphus), considerando como positivosa prova detuberculina osqueapresentassemaumentona espessuradapele em pelo menos $2 \mathrm{~mm}$.
Os resultados encontrados neste experimento, a partir dos quais propõe-se um padrão de interpretação para o teste tuberculínico (intradérmico simples na região cervical eo cervical comparativo) realizado em ovinos, vem ao encontro dessas necessidades, representando importanteferramenta a ser utilizada no controle da tuberculose.

Os valores padrões recomendados pelo BRASIL (2004a) no PN CEBT, pararealizar aleituraeinterpretação dos resultados obtidos no TCC em bovinos apresentam medidas limites para positividade que são superiores às estabel ecidas para os ovinos neste experimento. Assim, asimplesutilização destesvalores de bovinos para ovinos determinaria uma falsa negatividade de ovinos à presença da tuberculose, invalidandoo uso desses padrões deinterpretação do testetuberculínico nesses pequenos ruminantes. Tal fato também pode ser verificado quando se consideram os valores padrões de interpretação estabelecidos para caprinos porSILVA (2004) eSILVA et al. (2006), osquaisapresentam-seinferioresàquel es debovinos. Esses autores consideram osanimais como positivos quando o aumento da espessura da pelefor igual ou superior a 2,50 $\mathrm{mm}$, tendo assim uma pequena diferença em relação aos valores padrões encontrados neste experimento para ovinos (animais positivos quando o aumento da espessura da pelefor igual ou superior a $2 \mathrm{~mm}$ ), indicando não ser recomendável a utilização dos val ores deinterpretação debovinosou de caprinos para a realização do teste tuberculínico nos ovinos.

\section{CONCLUSÕES}

Com base nos resultados encontrados no experimento, propõe-se que, no teste intradérmico simples, na região cervical, realizado em ovinos, a reação à tuberculina bovina seja considerada: negativa, quando apresentar um aumento menor que $1 \mathrm{~mm}$; inconclusiva, quandooaumentosituar-seentrele2,40 $\mathrm{mm}$, e positiva quando for igual ou maior a $2,40 \mathrm{~mm}$.

Para o teste cervical comparativo, a reação à tuberculina bovina deve ser considerada: negativa, quandooaumentodaespessuradepel efor menor que à aviária ou maior em até $0,90 \mathrm{~mm}$; inconclusiva, quando for maior queaquelaà aviária comaumentos entre 1 e 1,90 $\mathrm{mm}$, e positiva quando for superior a aviaria em pelo menos $2 \mathrm{~mm}$.

Os valores recomendados pelo PNCEBT - BRASIL (2004a) para a leitura e interpretação do teste tuberculínico cervical comparativo em bovinos e bubalinos e os valores recomendados para caprinos não devem ser aplicados aos ovinos.

A resposta dos ovinos ao teste de tuberculina foi maisintensa em animaissensibilizados peloM . bovis 
quando comparadas ao grupo sensi bilizado pelo M . avium. O aumentodaespessuradapeleinduzidopela tuberculinabovinanosovinossensibilizadospeloM. bovis foi, em média, duas vezes superior ao aumento induzido pela tuberculina aviária.

\section{REFERÊNCIAS}

AlLen, G.M. Tuberculosis in sheep - a very rare disease. Surveillance, v.15, n.5, p.8-9, 1988.

Antunes, J.L.F.; M orais, M. de; Biazevic, M.G.H.; Waldman, E.A.; CorrêA, M.O.A. Tuberculose e leite: elementos para a história de uma polêmica. História, Ciência, Saúde M anguinhos, v.9, n.3, p.609-629, 2002.

Barton, M.D.; AcLand, H.M. Mycobacterium avium serotype 2 infection in a sheep. A ustralian $V$ eterinary Journal, v.49, n.4, p.212-213, 1973.

BenCINI, R.; PuLINA, G. Thequality of sheep milk: areview. A ustralian Journal of Experimental A griculture, v.37, n.4, p.485-504, 1997.

Benesi, F.J.; Pinheiro, S.R.; Maiorka, P.C.; Benites, N.R.; BirgeL JunIOR, E.H.; GreGory, L. Tuberculosisin goat in Brazil: case report. In: WORLD BUIATRICS CONGRESS, 24., 2006, Nice, Fra. Proceedings. 1 CDROM.

BraSIL. MinistériodaAgriculturaPecuáriaeA bastecimento. Secretaria de Defesa Agropecuária. Instrução N ormativa SD A no 6, de 8 dejaneiro de 2004 a. A prova 0 Regulamento Técnico do Programa $\mathrm{N}$ acional de Controlee Erradicação da Brucel oseet uberculose A nimal. Disponível em: বhttp:/ / www.agricultura.gov.br/ >. A cesso em: 10 jan. 2005.

BrasIL. MinistériodaAgriculturaPecuáriaeA bastecimento. Secretaria de Defesa Agropecuária. Instrução $\mathrm{N}$ ormativaSD A $\mathrm{n}-87$, del0 dedezembrode2004b. A prova o Regulamento Técnico do Programa $\mathrm{N}$ acional deSanidade dos Caprinos eO vinos (PN SCO). Disponível em: <http:/ / www.agricultura.gov.br/ >. A cesso em: 10jan. 2005.

Brito, M.A.;GonzÁlez,F.D.;RIBEIRo,L.A.;CAMpos, R.; LACERDA, L.; BARbosa, P.R.; Bergmann, G.Composição do sangue e do leite em ovinos leiteiros do sul do Brasil: variações nagestação enalactação.CiênciaR ural, v.36, n.3, p.942-948, 2006.

BRITo, M. A.V ariação dos perfis metabólico, hematológico elácteo de ovinos leiteiros em confinamento. 2004. 59f. Dissertação (Mestrado em PatobiologiaA nimal). - Faculdade de Medicina Veterinária, Universidade Federal do Rio Grande do Sul, Porto A legre, 2004.

Cordes, D.O.; Bullians, J.A.; lake, D.E.; Carter, M.E. Observations on tuberculosis caused by mycobacterium bovis in sheep. $\mathrm{N}$ ew $\mathrm{Z}$ eal and Veterinary Journal, v.29, n.4, p.60-62, 1981.

Corrin, K.C.; CARTer, C.E.; KISSLING, R.C.; LISLE, G.W. Short Interval intradermal skin testing in farmed red deer (Cervus elaphus) inoculated with $M$ ycobacterium bovis. $\mathrm{N}$ ew Z eal and V eterinary Journal, v.35, n.12, p.204-207, 1987.

Craig, J.F.; Davies, G.O. Tuberculosis in sheep. Veterinary Record, v.50, n.36, p.1156-1157, 1938.
CREECH, G.T. Bovinetypeof tuberculosisin sheep.A merican Journal V eterinary Research, v.1/ 2, n.1, p.23-25, 1940.

DaVIDSON, R.M.; AlLeY, M.R.; BeatSON, N.S. Tuberculosis in a flock of sheep. N ew Z eal and $V$ eterinary J ournal, v.29, n.1/ 2, p.1-2, 1981.

Duffield, B.J.; N orton , J.H.;Streeten, T.A.A pplication of the comparativecervical test to theidentification of fal se positive reactions to the bovine tuberculin caudal fold test. A ustralian Veterinary Journal, v.62, n.12, p.424-426, 1985.

FOULERTON, A.G.R.A caseof tuberculosisin a sheep.journal of Comparative Pathology and Therapeutics, v.15, p.102104, 1902.

González Llamazares, O.R.; GutírRrez Martin, C.B.; A lvarez Nistal,D;;Puente Redondo,V.A.;D ominguez Rodriguez, L.; Rodriguez Ferri, E. F. Field evaluation of thesingle intradermal cervical tuberculin test and the interferon-gammaassay for detection and eradication of bovine tuberculosis in Spain. Veterinary M icrobiology, v.70, p.55-66, 1999.

GraphPAd SOFTWARE. Instat guide: www.graphpad.com. Cary: Graphpad Software,1998. 1CD-Rom.

GRIFFITH, A.S. Tuberculosis of the domesticated species of animals. Journal of Comparative Pathology, v.41, p.109127, 1928.

HIEPE, H.T.Enfermidades del a oveja.Zaragoza: Acribia, 1972. 391p.

JeNSEn, R.; SWIF, B. L. Diseases of sheep. 2. ed. Philadel phia: Lea \& Febiger, 1982. 330p. Diseases of the respiratory system

LESSLIE, I.W.; H EBERTY, C.N.Comparasion of thespecificity of human and bovine tuberculin PPD for testing cattle. 3- National trial in Great Britan. V eterinary Record, v.96, p.338-341, 1975.

LukE, D. Tuberculosis in the horse, pig, sheep and goat. V eterinary Record, v.70, n.26, p.529-535, 1958.

M'FADYEAN, J. A case of Tuberculosis in a sheep. Journal of ComparativeP athology and Therapeutics, v.15, p.158-159, 1902.

MACKInTOSH, C.G.; LISLE, G.W.; Collins, D.M.; Grifin, J.F.T. Mycobacterium diseases of deer. $\mathrm{N}$ ew Z ealand Veterinary Journal, v.52, n.4, p.163-174, 2004.

Malone, F.E.; Wilson, E.C.; Pollock, J.M.; Squce, R.A. Investigations into an aoutbreak of tuberculosis in a flock of sheep in contact with tuberculous cattle, J ournal of V eterinary M edicine. Series B , v.50, p.500-504, 2003.

Marsh, H. Newsom's Sheep diseases. 3. ed. Baltimore: Williams \& Wilkins, 1965. 456p.

MuRPhY, J.M. Caseof tuberculosis (bovinetype) in asheep in Ireland. Veterinary Record, v.15, n.49, p.1488-1489, 1935.

Peeters, R.; Buys, N.; Robijns, L.; Vanmontfort, D.; Van ISTERDAEL, J. Milk yield and milk composition of Flemish Milksheep, Suffolk and Texel ewes and their crossbreds. Small Ruminant Research, v.7, p.279-288, 1992.

Pugh, D.G.Clínica deovinos ecaprinos. SãoPaulo: Roca, 2005. 513p.

Radostits, O.M.; Gay, C.C.; BLoOd, D.C.; HinchCliff, K.W. Clínica V eterinária - um tratado de doenças de bovinos, 
ovinos, suínos, caprinos e eqüinos. 9. ed. Rio de Janeiro: Guanabara Koogan, 2002. 1737p.

Ragassa, A.Y.; AmENI, G. Sinsitivity and especificity of a singleintradermal tuberculin test at thecervical and caudal folds in zebu cattle in Ethiopia. Indian J ournal of A nimal Sciences, v.71, n.4, p.325-327, 2001.

Riet-CorrêA,F.;Schild,A.L.;M endez,M.D.C.; L emos, R.A.A. $D$ oenças de ruminantes e eqüinos. São Paulo: Varela, 2001. v.1, 425p.

RoBinson, E.M. Tuberculosis in sheep and goats. Journal South A frica V eterinary M edical A ssociation, v.26, n.2, p.95-104, 1955.

Roxo, E.; Vasconcellos, S.A.; Pinheiro, S.R.; Baruselli, P.S.; MACRUZ,R.; L EITE, C.Q.L. Evaluation of tuberculinskin reaction in buffaloes (Bubalus bubalis). A rquivos do Instituto Biológico, São Paulo, v.65, n.1, p.81-92, 1998.

SAноо, B. N. Tuberculosis and tuberculin with special reference to PPD tuberculin- a review. Indian V eterinary Journal, v.27, n.5, p.319-331, 1951.

SA MPAIO, I.B.M. Estatística aplicada à experimentação animal. 2. ed. Belo Horizonte: Fundação de Ensino e Pesquisa em Medicina Veterinária e Zootecnia, 2002. $265 \mathrm{p}$.
SiLva, P.E.G. Padronização do alergoteste da tuberculina em caprinos (Caprahircus). 2004. 73f.Dissertação(Mestrado em Clínica Médica). - FaculdadedeM edicina Veterinária e Zootecnia, Universidade de São Paulo, São Paulo, 2004.

Silva, P.E.G.; Pinheiro, S.R.; Leal, M.L.R.; Bertagnon , H.G.; MotTA, P.M.P.C.; SNhorini, I.L.; VAsconcellos, S.A.; BENESI, F.J. Teste de tuberculinização em caprinos (Capra hircus) experimentalmente sensibilizados. Ciência Rural , v.36, n.3, p.880-886, 2006.

Suther, B.E.; Franti, C.E.; Page, H.H. Evaluation of a comparativeintradermal tuberculintestin California dairy cattle. A merican Journal of V eterinary Research, v.35, n.3, p.379-387, 1974.

Thoen, C.O.Tuberculosis. J ournal of the A merican $V$ eterinary M edical A ssociation, v.193, n.9, p.1045-1048, 1988.

TIZARD, I.R. Introdução a imunologia veterinária. 6. ed. São Paulo: Roca, 2002. 532p.

WнIтTY,B.T.;D EмPSEY , D.General ised tuberculosisinasheep. Irish V eterinary Journal, v.28, n.11, p.241-242, 1974.

Recebido em 15/ 1/ 07

A ceito em 28/ 7/ 07 